\title{
Hohe Ansprechraten mit ALK-Inhibitor Crizotinib
}

\begin{abstract}
Patienten mit fortgeschrittenem nichtkleinzelligem Lungenkarzinom (NSCLC), die eine spezifische Genveränderung, ein Rearrangement des ALK-Gens, aufweisen, können von der Therapie mit dem ALK-Inhibitor Crizotinib profitieren. Fast $90 \%$ der Patienten erreichten in einer erweiterten Phase-I-Studie mit Crizotinib (250mg 2-mal täglich oral) mindestens eine Krankheitsstabilisierung, bei mehr als der Hälfte der Patienten wurde eine Reduktion der Tumorgröße festgestellt (Bang Y et al., ASCO 2010: \#3).
\end{abstract}

In der Auswertung der noch nicht abgeschlossenen erweiterten Phase-I-Studie wurden die Daten von 82 Patienten mit ALKpositivem NSCLC berücksichtigt. Die meisten Patienten hatten ein Adenokarzinom (96\%) und waren Nie- (76\%) oder ehemalige Raucher (23\%). 41\% der Patienten hatten zuvor bereits mindestens 3 Therapien erhalten, $18 \%$ der Patienten waren nach 2 Vortherapien und 33\% der Patienten nach 1 Vortherapie in die Studie eingeschlossen worden.

Nach einer medianen Behandlungsdauer von 5,7 Monaten betrug die objektive Ansprechrate (ORR [objective response rate]) 57\% (95-\%-Konfidenzintervall [95-\%KI] 46\%-68\%). Die Tabelle zeigt die objektive Ansprechrate in Abhängigkeit von der Anzahl der Vortherapien. Die Dauer des Therapieansprechens betrug 1 bis 15 Monate. 87\% der Patienten erreichten zu Woche 8 mindestens eine Stabilisierung der Erkrankung (DCR [disease control rate: komplettes und partielles Ansprechen plus Krankheitsstabilisierung]; 95-\%-KI 77\%-93\%). Das mediane progressionsfreie Überleben (PFS) war zum Auswertungszeitpunkt noch nicht erreicht. Die Wahrscheinlichkeit für ein PFS nach 6 Monaten betrug 72\%. Die Verträglichkeit der Therapie war laut Bang gut. Die häufigsten unerwünschten Ereignisse waren leichte bis mäßige gastrointestinale Ereignisse und visuelle Störungen.

Bang sieht in der Therapie mit Crizotinib einen potenziellen neuen Standard für die Behandlung von Patienten mit ALK-positivem NSCLC. Crizotinib wird derzeit in der PhaseIII-Studie PROFILE 1007 (NCT00932893) weiter untersucht. Martin J. Edelman, Baltimore/USA, hinterfragte in der Diskussion der Studienergebnisse die Notwendigkeit einer Phase-III-Studie, da Patienten mit ALK-positivem Tumor nur schlecht auf eine zytostatische Chemotherapie ansprechen und für die Therapie mit Crizotinib bei diesen Patienten, trotz intensiver Vortherapie, hohe Ansprechraten und ein dauerhaftes progressionsfreies Überleben gezeigt werden konnten.

\section{Personalisierte Therapie bei \\ Patienten mit NSCLC}

Bang zufolge unterstreichen die bislang vorliegenden Daten zu Crizotinib die Bedeutung der personalisierten Therapie bei
NSCLC-Patienten. Am Beispiel von Crizotinib kann eine schnelle Entwicklung von der Identifizierung der Zielstruktur bis zu ersten klinischen Studien gezeigt werden, so Bang. Im Jahr 2007 wurde die Bedeutung des ALK-Gen-Rearrangements, bei dem das EML4-ALK-Fusionsgen als wichtiges Onkogen beim NSCLC entsteht, beschrieben (Soda M et al., 2007, Nature 448: 561566). Dieses Onkogen führt zur Expression eines tumorspezifischen, aktivierten, für das Wachstum und die Entwicklung von Tumorzellen zentralen Proteins, der Anaplastic Lymphoma Kinase (ALK). Die Genveränderung liegt bei schätzungsweise $3 \%$ bis 5\% der NSCLC-Patienten vor, sie kann u.a. mittels FISH (Fluoreszenz-in-situ-Hybridisierung) nachgewiesen werden (Shaw AT et al., 2009, J Clin Oncol 27: 4247-4253). AM

\section{Metastasiertes kolorektales Karzinom - KRAS-Wildtyp-Tumoren}

\section{Nutzen der First-line-Therapie mit Cetuximab bestätigt}

Die zusätzliche Gabe von Cetuximab zur First-line-Chemotherapie verbesserte bei Patienten mit metastasiertem kolorektalem Karzinom ( $\mathrm{mCRC}$ ) mit KRAS-Wildtyp-Tumor das beste Gesamtansprechen sowie das progressionsfreie und Gesamtüberleben signifikant $(p<0,0001, p<0,0001$ und $p=0,0002$ ). Das bestätigen die Ergebnisse einer Analyse der gepoolten Wirksamkeitsdaten der Studien CRYSTAL und OPUS in Abhängigkeit vom KRAS- und BRAF-Mutationsstatus (Bokemeyer C et al., ASCO 2010: \#3506).
Das beste Therapieergebnis erreichten Patienten mit KRAS- und BRAF-Wildtyp-Tumor. BRAF-Mutationen wurden bei 8,8\% der Patienten mit KRAS-Wildtyp-Tumor festgestellt. Sie scheinen ein Indikator für eine schlechte Prognose zu sein, so Bokemeyer. Dennoch könnten auch Patienten mit KRAS-Wildtyp-Tumor und BRAF-Mutationen noch einen Nutzen von der zusätzlichen Cetuximab-Gabe zur First-line-Chemotherapie haben. Den Analyseergebnissen zufolge ist der BRAF-Mutationsstatus kein relevanter prädiktiver Marker für den First-line-Einsatz von Cetuximab. $A M$ 